\title{
Low Voltage STEM for the Study of Defects in 2D Materials
}

\author{
Wu Zhou ${ }^{1}$, Jaekwang Lee ${ }^{2,1}$, Myron D. Kapetanakis ${ }^{2,1}$, Micah P. Prange ${ }^{2,1}$, Andrew R. Lupini ${ }^{1}$, \\ Sokrates T. Pantelides ${ }^{2,1}$, Juan-Carlos Idrobo ${ }^{1}$, Stephen J. Pennycook ${ }^{1,2}$ \\ 1. Materials Science \& Technology Division, Oak Ridge National Laboratory, Oak Ridge, TN \\ 37831, USA \\ 2. Department of Physics and Astronomy, Vanderbilt University, Nashville, TN 37235, USA
}

Two dimensional (2D) materials, such as graphene, h-BN and $\mathrm{MoS}_{2}$, have attracted significant attention in recent years due to their unique properties and promising applications. Of particular interests are the structural defects present in these 2D materials, including point defects, defect complexes, grain boundaries, and edges, as they can induce local changes to the material properties and provide exciting opportunities to create new functionalities [1]. Understanding the detailed atomic makeup of these defects and their behavior beyond the ground states is an important first step to correlate the defect structure with local properties in these 2D materials.

Aberration-corrected scanning transmission electron microscopy (STEM) at low voltage provides a feasible way to study the defect structure and measure the local properties with single atom sensitivity via imaging and spectroscopic imaging [2]. By reducing the electron beam energy and dose, some of the defects could remain stable during imaging, which allows reliable analysis of the defect structure. By increasing the electron beam energy or dose, defects can be deliberately generated, and their dynamics can be studied via sequential imaging.

Figure 1 shows annular dark-field (ADF) images of 3- and 4-fold coordinated Si substitutional impurities in graphene, constructed via cross-correlation of multiple low-dose images taken at 60 $\mathrm{kV}$. This image acquisition scheme can help to minimize electron-beam-induced structural modification, improve the signal-to-noise $(\mathrm{S} / \mathrm{N})$ ratio and increase the accuracy of atomic position measurements [2]. By optimizing the electron dose, Si L-edge fine structures from the individual $\mathrm{Si}$ atoms with good $\mathrm{S} / \mathrm{N}$ ratio can be obtained via electron energy loss (EEL) spectrum imaging, allowing direct determination of the nature of the chemical bonding from single impurity atoms in graphene [3]. We will also show that using low-dose, low-voltage, STEM imaging, distinct grain boundary structures in various 2D materials can be directly identified.

A sequence of $\mathrm{ADF}$ images from an embedded $\mathrm{Si}_{6}$ cluster in a graphene nano-pore is shown in Figure 2. In this case, the electron dose is controlled to a level such that the energy transfer from the electron beam is sufficient to excite the reversible dynamics without destroying the structure. The structural information obtained from such sequential imaging can be used as important input for density functional theory calculations to explore the three-dimensional stable/meta-stable configuration of defect complexes and to study defect dynamics at the atomic level [4]. We will also show that for semiconducting or insulating 2D materials, where both knock-on and ionization damages can be significant, sequential imaging can also be used to explore the optimum electron beam energy and dose level for microscopy analysis. [5]

References:

[1] D S L Abergel et al, Advances in Physics 59 (2010), p. 261. 
[2] W Zhou et al, Microscopy and Microanalysis 18 (2012), p.1342.

[3] W Zhou et al, Physical Review Letters 109 (2012), p. 206803.

[4] J Lee et al, Nature Communications. in press (2013).

[5] This research was supported by NSF grant No. DMR-0938330 and a Wigner Fellowship through the Laboratory Directed Research and Development Program of Oak Ridge National Laboratory, managed by UT-Battelle, LLC, for the U.S. DOE (WZ); Oak Ridge National Laboratory's ShaRE User Facility program (JCI), which is sponsored by the Office of Basic Energy Sciences, U.S. DOE; Office of Basic Energy Sciences, Materials Sciences and Engineering Division, U.S. DOE (SJP, JL, ARL, STP); DOE grant DE-FG02-09ER46554 (MDK, MPP, STP).
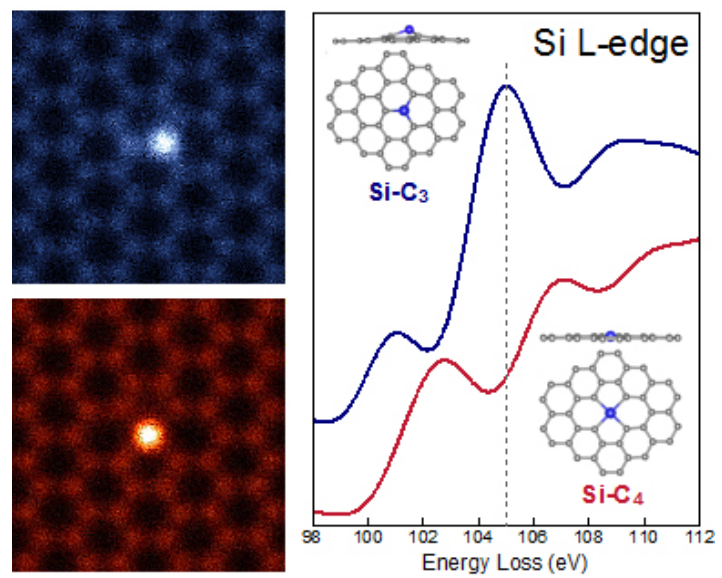

Fig. 1. (Left) ADF images of 3- and 4-fold coordinated Si atoms in graphene, and their respective Si L-edge fine structure extracted from spectrum images (Right). The combination of $\mathrm{ADF}$ and EEL spectrum imaging, with first-principles calculations, allows direct determination of the bonding configuration of the Si impurities. Reproduced from Ref [3].
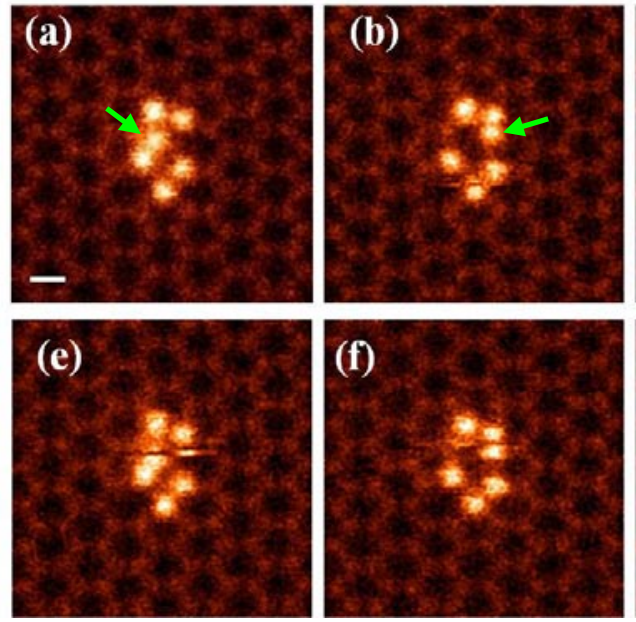
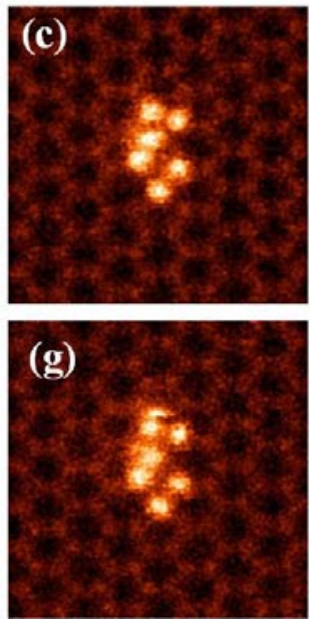
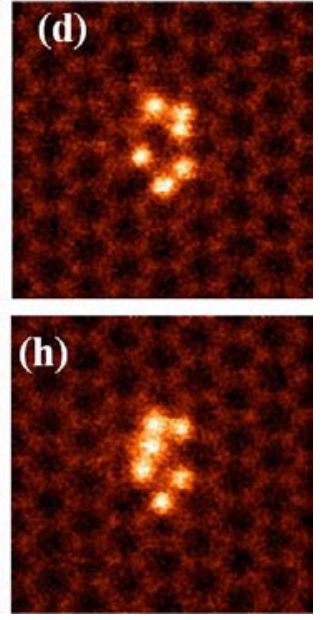

Fig. 2. Sequential ADF images of a $\mathrm{Si}_{6}$ cluster embedded in a graphene pore. By controlling the electron dose, it is possible to excite reversible dynamics of the embedded Si cluster with one of the six Si atoms (highlighted) jumping between the left and right configurations. Reproduced from Ref [4]. 